\title{
A new hybrid GRASP with the pilot method for the delay-constrained multicast routing problem
}

\author{
Ying Xu, Xiongfei Zheng, Renfa Li \\ College of Information Science and Engineering, Hunan University, Changsha \\ Hunan, 410082, CHINA \\ e-mail: hnxy@hnu.edu.cn,447236402@qq.com, lirenfa@vip.sina.com
}

\begin{abstract}
Multicast routing problem is a well know optimization problem for transmitting real-time multimedia applications in telecommunication networks. As the underpinning mathematical model, the constrained minimum Steiner tree problem in graphs is a well-known NP-complete problem. In this paper we investigate a new hybrid GRASP (Greedy Randomized Adaptive Search Procedure) approach where a pilot method is applied to further enhance the search for the Delay-Constrained Least-Cost (DCLC) multicast routing problem. Experimental results demonstrate the efficiency of the hybrid GRASP algorithm and the contributions of the post-processing pilot method to better solutions in most cases. The proposed GRASP approach is a competitive approach in solving the DCLC multicast routing problem.
\end{abstract}

Keywords- GRASP(Greedy Randomised Adaptive Search Procedure); Pilot Method; Multicast Routing

\section{INTRODUCTION}

Multicast routing is a well know technique which transfers information from a source to a group of destinations simultaneously. The rapid development of numerous multicast network applications (e.g. E-learning, E-commerce, video-conferencing) promotes the requirement multicast routing with certain QoS (Quality of Service) constraints in the underlying computer networks. Different application may have different QoS requirements, such as the cost of the transmission, the end-to-end delay from the sender to destinations, the bandwidth consumption, the delay jitter, the packet lost ratio and the transmission hop count, etc. Multicast QoS routing has received significant research attention in the area of computer networks and algorithmic network theory [1-3]. This paper concerns two of the most important QoS demands, the total cost of the edges in the multicast tree from the source to all the destinations and the end-to-end delay bound for the total delay from the source to any destination in the multicast group.

Multicast routing problems can be modeled as a Minimum Steiner Tree Problem in Graphs (MStTG)[4]. Given an undirected graph $G=(V, E)$, where $V$ is a set of nodes, $E$ is a set of edges, and a subset of nodes $D \subseteq V$, a Steiner tree is a tree which connects all the nodes in $D$ using a subset of edges in E. Extra nodes in $W D$ may be added to the Steiner tree, called the Steiner nodes. The objective of a MStTG problem is to search a minimal Steiner tree with respect to the total edge costs $c(e), e \in E$, which has been proven to be NP-complete [5]. The Delay-Constrained Least-Cost (DCLC) multicast routing problem can be seen as a delay-constrained Steiner tree, which is also NPcomplete [6-8].

In this paper, we investigate a new hybrid GRASP approach by applying a pilot method as the post-processing technique to further improve the search for the DCLC multicast routing problem. To our knowledge, very little attention has been given to the GRASP approach on multicast routing and we know only one exception in [9]. In our previous work, a GRASP with variable neighborhood search approach, namely GRASP-VND, has been proposed and successfully applied to solve the DCLC multicast routing problem [10]. Motivated by the good performance of GRASP-VND, in this paper, we incorporate the idea of applying a pilot method, named GRASP-VND+pilot, to improve the performance of the hybrid GRASP approach. We test the proposed hybrid GRASP algorithm on a set of benchmark Steiner tree problems in the OR library[11] and a group of random graphs. Computational results indicate that GRASP-VND+pilot leads to better results compared with GRASP-VND. In addition, it outperforms other two algorithms, namely a multi-start algorithm of an extended VND search algorithm [12] and the GRASP-CST algorithm [9]. Our proposed GRASP-VND+pilot algorithm has the overall best performance in terms of the average tree cost in comparison with the existing algorithms and heuristics.

The rest of the paper is organized as follows. In section II, we present the problem definition and related work. Section III presents the proposed GRASP-VND+pilot algorithm. We evaluate our algorithm by computer simulations and summarize the obtained simulation results in section IV. Finally, section V concludes this paper and presents the possible future work.

\section{THE PROBLEM DEFINITION AND RELATED WORK}

\section{A. The network model and problem definition}

We define a computer network as a connected, directed graph $G=(V, E)$ with $|V|=n$ nodes and $|E|=l$ links, where $V$ is a set of nodes and $E$ is a set of links. For each link $e=(i, j) \in E$, link cost $c(e)$ and link delay $d(e)$ are defined. $c(e)$ is associated with the utilization of the 
corresponding link's resources. $d(e)$ is related the transmission delay of messages along the link. The computer network is asymmetric, i.e., for link $e=(i, j)$ and link $e^{\prime}=(j, i)$, it is possible that $c(e) \neq c\left(e^{\prime}\right)$ and $d(e) \neq d\left(e^{\prime}\right)$. A multicast routing problem includes a source node $s$ and a set of destination nodes $D$ called multicast groups, which receive data stream from the source, denoted by $D \subseteq V \backslash\{s\}$.

A path from node $u$ to $v$ is an ordered set of links, denoted by $P(u, v)=\{(u, i),(i, j), \ldots,(k, v)\}$. A multicast tree $T(s, D) \subseteq E$ is a tree rooted at source $s$ and spanning all destination nodes in $D . P\left(s, r_{i}\right) \subseteq T$ represents the path from $s$ to $r_{i} \in D$ in $T$. The sum of the delays of all links along $P\left(s, r_{i}\right)$ is the end-to-end delay from $s$ to each destination $r_{i}$ which is defined as

$$
\operatorname{Delay}\left(r_{i}\right)=\sum_{e \in P\left(s, r_{i}\right)} d(e), \forall r_{i} \in D
$$

$\operatorname{Delay}(T)$ represents the delay of tree $T$, which is the maximum delay among all $\operatorname{Delay}\left(r_{i}\right)$ from source to each destination, i.e.

$$
\operatorname{Delay}(T)=\max \left\{\operatorname{Delay}\left(r_{i}\right) \mid \forall r_{i} \in D\right\}
$$

$\operatorname{Cost}(T)$ is defined as the cost of tree $T$, which is the sum of the cost of all links in the tree, i.e.

$$
\operatorname{Cost}(T)=\sum_{e \in T} c(e)
$$

Real world applications can only tolerant a bounded transmitting delay from source to each destination. In this paper, we assume that the delay bound $\Delta=\delta_{i}$ for all destinations.

Given these definitions, we formally define the DelayConstrained Least-Cost (DCLC) multicast routing problem as:

The DCLC Multicast Routing Problem: Given a network $G$, a source node $s$, a destination node set $D$, a link cost function $c(\cdot)$, a link delay function $d(\cdot)$, and a delay bound $\Delta$, the objective of the DCST Problem is to construct a multicast tree $T(s, D)$ such that the delay bound is satisfied, and the tree cost $\operatorname{Cost}(T)$ is minimized. We define the objective function as:

$$
\min \left\{\operatorname{Cost}(T) \mid P\left(s, r_{i}\right) \subseteq T(s, D), \operatorname{Delay}\left(r_{i}\right) \leq \Delta, \forall r_{i} \in D\right\}
$$

\section{B. Related work}

The multicast routing problem has received extensive studies, and consequently many exact and heuristic algorithms have been developed. Most of these algorithms can be classified as source-based or destination-based multicast routing algorithms. In source-based algorithms, each node has all the necessary information to construct the multicast tree [13-18]. While destination-based algorithms do not require that each node maintains the entire network status information, and multiple nodes participate in constructing the multicast tree $[6,14,19,20]$.
In recent years, meta-heuristic algorithms such as simulated annealing [21,22], genetic algorithm [23,24], tabu search [25-28], GRASP [9,10], path relinking [29], VNS [12], scatter search [30] and particle swarm optimization [31] have been investigated for various multicast routing problems. A GRASP meta-heuristic hybridized with VNDMR [12], namely GRASP-VND, is proposed in our previous work in [10], which has showed good performance for the solving the DCLC multicast routing problem.

It can be seen from the literature review, although GRASP is an efficient meta-heuristic for optimization problems, little attention has been given to GRASP for solving the multicast QoS routing problem. Motivated by the successful applications of the pilot method in the literature for the Steiner tree problem in Graphs in [32] and the network design problem in [33], we further study a pilot method as a post optimization procedure to improve the performance of our previous GRASP-VND. Our aim is to investigate advanced hybrid meta-heuristics and towards designing more efficient approaches for the DCLC multicast routing problem.

\section{THE PROPOSED HYBRID GRASP APPROACH}

GRASP (Greedy Randomized Adaptive Search Procedure) is an efficient multi-start meta-heuristic for a wide range of optimization problems [34]. An iteration of GRASP consists of two phases: a construction phase which is used to generate a feasible solution, and a local search phase which is applied to explore the neighborhood of the feasible solution until a local minimum is found. GRASP builds the feasible solution by iteratively creating a candidate list of elements, called the restricted candidate list (RCL), and by evaluating the elements not yet included in the partial solution with a certain greedy function. To further improve the feasible solution generated in the construction phase, a local search is applied to search for better neighboring solutions of the feasible solutions. After a given number of iterations, the best overall solution is kept as the final solution. More detailed descriptions of the GRASP heuristic can be found in $[34,35]$.

GRASP meta-heuristic is effective and easy to implement and few parameters need to be set and tuned, thus it has been successfully applied to solve a wide range of combinatorial optimization problems [36-38]. Our motivation is to investigate effectiveness a pilot method as the post optimization process to the performance of the hybrid GRASP approach for the DCLC multicast routing problem.

\section{A. The construction phase}

In the construction phase of our GRASP-VND+pilot algorithm, we use the same greedy randomized procedure as that in [10] to create the randomized initial solution, which is adopted from the greedy strategy in [9]. The construction phase firstly calculates the shortest path from source to each destination. A parameter a in GRASP is used to select the candidate paths to be included in the RCL. 


\section{B. The local search phase}

First, confirm that you have the correct template for your paper size. This template has been tailored for output on the US-letter paper size. If you are using A4-sized paper, please close this template and download the file for A4 paper format called "CPS_A4_format".

\section{The application of the pilot method}

The pilot method may be seen as an intelligent technique to look ahead of possible choices after certain iterations to support better final decision of the options concerned. It is a tempered greedy method by performing repetition of heuristics in order to record the best result before getting to a promising solution. Pilot methods have been investigated for solving various combinatorial optimization problems [32, $33,40-42]$, in which some are under different names, for example the rollout method in [41] and [42].

We propose the GRASP-VND+pilot algorithm which is an extension of our GRASP-VND in [10] by applying a pilot method to explore better neighboring solutions after a local optimum has been found by the local search phase. The pilot method is used as an enhancement mechanism, where each pilot includes two procedures:

1) Shaking process: During the shaking process, a destination node is randomly selected. Then, a set of back up paths which connect the source and the destination node is generated for the chosen destination by using the $k$-th shortest path algorithm [43]. The pilot shakes the current solution by choosing one path at random from the back up path set to replace the original path from the source to the destination node in the current tree.

2) Pilot search procedure: Each pilot searches better solutions by looking ahead more neighboring solutions defined by the pilot heuristic in the pilot search procedure. Our pilot heuristic uses the same operation as the node-based neighborhood structure in VNDMR which is easy to implement and has shown to be effective. The pilot search procedure repeats until no further improvement can be achieved.

In the pilot method, a key factor is the pilot depth, which determines the actual search depth of the pilot method, i.e. the quality of the results and the runtime of the search. In our pilot method, the pilot depth is a given number of iterations of the pilot heuristic. A larger pilot depth may lead to better solutions; however, it usually means longer runtime.

\section{PERFormance EVAluations}

We use a multicast routing simulator (MRSIM) implemented in $\mathrm{C}++$ based on Salama's generator [1] to generate random network topologies. More details description of the simulator can be found in our previous work in [10]. All simulations were run on a Windows XP computer with PVI 3.4GHZ, 1G RAM.

In order to compare the performance of GRASPVND+pilot with other existing algorithms, we tested
GRASP-VND+pilot on a group of randomly generated graphs which has been used as benchmark test instances in $[10,12,30,31]$. After a number of initial tests, the number of iterations is set as 4 , a (the parameter for creating RCL) is set as 5 in both GRASP-VND and GRASP-VND+pilot. The pilot depth is set as $t=5$.

TABle 1. COMPARISON OF THE AVERAGE TREE COST OBTAINED BY GRASP-VND+PILOT AND SOME EXISTING ALGORITHMS ON THE RANDOM GRAPHS

\begin{tabular}{ccc}
\hline & Algorithms & $\begin{array}{c}\text { Average } \\
\text { Tree Cost }\end{array}$ \\
\hline Heuristics & KPP1 [13] & 905.581 \\
& KPP2 [14] & 911.684 \\
& BSMA[18] & 872.681 \\
\hline GA-based & Wang et al. [23] & 815.969 \\
Algorithms & Haghighat et al. [24] & 808.406 \\
\hline & Skorin-Kapov and Kos[26] & 897.875 \\
TS-based & Youssef et al. [25] & 854.839 \\
Algorithms & Wang et al. [21] & 869.291 \\
& Ghaboosi and Haghighat [28] & 739.095 \\
\hline Path relinking & Ghaboosi and Haghighat [29] & 691.434 \\
\hline VNS & VNDMR [12] & 658.967 \\
Algorithms & Multi-VND [10] & 656.777 \\
\hline Scatter Search & SSPR-VND [30] & 644.840 \\
Algorithm & JPSO [31] & 662.100 \\
\hline PSO & GRASP-CST [9] & 669.927 \\
Algorithm & GRASP-VND [10] & 654.520 \\
\hline GRASP & GRASP-VND+pilot & 650.823 \\
\hline Algorithms &
\end{tabular}

TABle 2. AVERAGE TREE COST, STANDARD DEVIATION OF THE TREE COST AND EXECUTION TIME OF GRASP-VND+PILOT, SSPR-VND, GRASP-VND AND MULTI-VND ON RANDOM GRAPHS.

\begin{tabular}{|c|c|c|c|c|c|c|}
\hline \multirow{3}{*}{$\begin{array}{l}\text { Network } \\
\text { Size }\end{array}$} & \multicolumn{6}{|c|}{ Algorithms } \\
\hline & \multicolumn{3}{|c|}{ GRASP-VND+pilot } & \multicolumn{3}{|c|}{$S S P R-V N D$} \\
\hline & Cost & $\sigma$ & $\begin{array}{c}\text { Time } \\
(\mathrm{s})\end{array}$ & Cost & $\sigma$ & $\begin{array}{c}\text { Time } \\
\text { (s) }\end{array}$ \\
\hline 10 & 94.7 & 0 & 0.02 & 94.7 & 0 & 0.04 \\
\hline 20 & 271.1 & 1.48 & 0.15 & 272.5 & 2.41 & 0.31 \\
\hline 30 & 392.3 & 0 & 0.61 & 393.5 & 3.57 & 1.45 \\
\hline 40 & 513.6 & 0.35 & 1.59 & 513.3 & 0.00 & 3.52 \\
\hline 50 & 663.8 & 6.75 & 3.42 & 660.8 & 0.53 & 9.58 \\
\hline 60 & 754.8 & 7.08 & 6.93 & 748.1 & 7.03 & 13.64 \\
\hline 70 & 780.1 & 3.94 & 13.84 & 779.5 & 5.97 & 29.61 \\
\hline 80 & 878.9 & 16.55 & 31.19 & 863.3 & 5.93 & 66.36 \\
\hline 90 & 1163.7 & 28.67 & 52.20 & 1132 & 19.35 & 116.42 \\
\hline 100 & 995.0 & 5.22 & 58.38 & 989.8 & 3.19 & 177.45 \\
\hline Avg. & 650.8 & 7.001 & 16.83 & 644.8 & 4.8 & 41.84 \\
\hline
\end{tabular}

Table 1 shows that GRASP-VND+pilot performs better than GRASP-VND, Multi-VND and GRASP-CST in terms of the average tree cost, which demonstrates the effectiveness of the pilot method proposed in this paper. Table 2 compares the average tree cost, standard deviation and execution time of GRASP-VND+pilot with the best performed algorithm SSPR-VND[30] on each network size 
of these random graphs are given. We can see that SSPRVND consumed much longer average computational time (41.84 seconds) than that (16.83 seconds) of GRASPVND+pilot. GRASP-VND+pilot algorithm obtained so far the second best average tree cost compared with other existing algorithms which shows it is a competitive approach for solving the DCLC multicast routing problem.

\section{CONCLUSIONS}

In this paper, we investigated hybrid GRASP approaches for solving Delay-Constrained Least-Cost (DCLC) multicast routing problems. To guide the search to better solutions, a new hybrid GRASP-VND+pilot algorithm is proposed by applying a pilot method to further improve the quality of solutions. Experiment results show that the pilot method in GRASP-VND+pilot contributed to better results in most cases compared with previous GRASP-VND algorithm. Our GRASP-VNS+pilot algorithm obtained the second best performance comparing with existing algorithms in terms of average tree cost.

Some interesting future research directions could be explored. In reality, some networks such as wireless ad hoc networks, the topologies of networks are mostly dynamic with nodes leaving and joining the network at various times. The adaptation and extension of the hybrid GRASP approach to the problem of highly constrained and dynamic multicast routing deserve further research.

\section{ACKNOWLEDGMENT}

This research is supported by Natural Science Foundation of China (NSFC project No. 61202289) and the project of the support plan for young teachers in Hunan University, China (Ref. 531107021137).

\section{REFERENCES}

[1] H.F. Salama, D.S. Reeves, and Y. Viniotis, "Evaluation of multicast routing algorithms for real-time communication on high-speed networks," IEEE Journal on Selected Areas in Communications, Vol.15, No.3, 1997, pp. 332-345.

[2] C.K. Yeo, B.S. Lee, and M.H. Er, "A survey of application level multicast techniques," Computer Communications, Vol. 27, No.15, 2004, pp. 1547-1568.

[3] X. Masip-Bruin, M. Yannuzzi, and J. Domingo-Pascual, A. Fonte, M. Curado, E. Monteiro, F. Kuipers, P. Van Mieghem, S. Avallone, G. Ventre, P. Aranda-Gutierrez, M. Hollick, R. Steinmetz, L. Iannone, K. Salamatian, "Research challenges in QoS routing," Computer Communications, Vol. 29, No.5, 2006, pp. 563-581.

[4] X. Cheng, and D.Z. Du, (eds.), "Steiner Trees in Industry," Kluwer Academic Publishers, Dordrecht, Netherlands, 2001.

[5] M.R. Garey, and D.S. Johnson, "Computers and Intractability: A Guide to the Theory of NP-Completeness," W.H. Freeman and Company, New York, 1979.

[6] L. Guo, and I. Matta, "QDMR: An efficient QoS dependent multicast routing algorithm," Proc. of the 5th IEEE RealTime Technology and Applications Symposium, 1999, pp. 213-222.

[7] C. Diot, W. Dabbous, and J. Crowcroft, "Multipoint communication: a survey of protocols, functions, and mechanisms," IEEE Journal on Selected Areas in Communications, Vol. 15, No. 3, 1997, pp.277-290.
[8] C.A.S. Oliveira, and P.M. Pardalos, "A survey of combinatorial optimization problems in multicast routing," Computers \& Operations Research, Vol. 32, No. 8, 2005, pp. 1953-1981.

[9] N. Skorin-Kapov, and M. Kos, "A GRASP heuristic for the delayconstrained multicast routing problem," Telecommunication Systems. Vol. 32, No.1, 2006, pp.55-69.

[10] Y. Xu, and R. Qu, "A GRASP approach for the Delay-constrained Multicast routing problem," Proc. of the 4th Multidisplinary International Scheduling Conference (MISTA4), Dublin, Ireland, 2009.

[11] J.E. Beasle, "An SST-based algorithm for the Steiner problem in graphs," Networks, Vol.19, 1989, pp.1-16.

[12] R. Qu, Y. Xu, and G. Kendall, "A Variable Neighborhood Descent Search Algorithm for Delay-Constrained Least-Cost Multicast Routing," Proc. of Learning and Intelligent OptimizatioN (LION3), Trento, Italy, 2009.

[13] V.P. Kompella, J.C. Pasquale, and G.C.Polyzos, "Multicast routing for multimedia communication," IEEE/ACM Transactions on Networking, Vol.1, 1993, 286-292.

[14] V.P. Kompella, J.C. Pasquale, and G.C. Polyzos, "Two distributed algorithms for the constrained Steiner tree problem," Proc. of the 2nd International Conference on Computer Communications and Networking, 1993, pp.343-349.

[15] Q. Sun, and H. Langendoerfer, "Efficient multicast routing for delaysensitive applications," Proc. of the 2nd workshop on protocols for multimedia systems, 1995, pp. 452-458.

[16] Q. Sun, and H. Langendoerfer, "An efficient delay-constrained multicast routing algorithm," Technical Report, Internal Report, Institute of Operating Systems and Computer Networks, TU Braunschweig, Germany, 1997.

[17] R. Widyono, "The design and evaluation of routing algorithms for realtime channels," Technical Report, ICSI TR-94-024, International Computer Science Institute, U.C. Berkeley, 1994.

[18] Q. Zhu, M. Parsa, and J. J. Garcia-Luna-Aceves, "A source-based algorithm for delay-constrained minimum-cost multicasting," Proc. of the 14th Annual Joint Conference of the IEEE Computer and Communication (INFOCOM'95), IEEE Computer Society Press, Washington, DC, USA, 1995, pp. 377-385.

[19] A. Shaikh, and K. Shin, "Destination-driven routing for low-cost multicast," IEEE Journal on Selected Areas in Communications, Vol. 15, 1997, pp.373-381.

[20] X. Jia, "A distributed algorithm of delay-bounded multicast routing for multimedia applications in wide area networks," IEEE/ACM Transactions on Networking, Vol. 6, 1998, pp. 828-837.

[21] X.L. Wang, and Z. Jiang, "QoS multicast routing based on simulated annealing algorithm," Proc. international and applications, 2004, pp. 511-516.

[22] K. Zhang, H. Wang, and F.Y. Liu, "Distributed multicast routing for delay and delay variation-bounded Steiner tree using simulated annealing," Computer Communications, Vol. 28, No.11, 2005, pp.1356-1370.

[23] Z. Wang, B. Shi, and E. Zhao, "Bandwidth-delay-constrained leastcost multicast routing based on heuristic genetic algorithm," Computer communications, Vol. 24, 2001, pp.685-692.

[24] A.T. Haghighat, K. Faez, M. Dehghan, A. Mowlaei, and Y. Ghahremani, "GA-based heuristic algorithms for bandwidth-delayconstrained least-cost multicast routing," Computer Communications, Vol. 27, 2004, pp.111-127.

[25] H. Youssef, A. Al-Mulhem, S.M. Sait, and M.A. Tahir, "QoS-driven multicast tree generation using tabu search," Computer Communications, Vol.25, No.11-12, 2002, pp.1140-1149.

[26] N. Skorin-Kapov, and M. Kos, "The application of Steiner trees to delay constrained multicast routing: a tabu search approach," Proc. of the seventh international Conference on Telecommunications, Zagreb, Croatia, 2003. 
[27] H. Wang, J. Fang, H. Wang, and Y.M. Sun, "TSDLMRA: an efficient multicast routing algorithm based on tabu search," Journal of Network and Computer Applications, Vol.27, 2004, pp.77-90.

[28] N. Ghaboosi, and A.T. Haghighat, "A tabu search based algorithm for multicast routing with QoS constraints," Proc. of the 9th International Conference on Information Technology, 2006, pp. 18-21.

[29] N. Ghaboosi, A.T. Haghighat, "A path relinking approach for DelayConstrained Least-Cost Multicast routing problem," Proc. of the 19th International Conference on Tools with Artificial Intelligence, 2007, pp. 383-390.

[30] Y. Xu, R. Qu, "A Hybrid Scatter Search Meta-heuristic for Delayconstrained Multicast Routing Problems," Applied Intelligence, Vol.36, No.1, 2010, pp.229-241.

[31] R. Qu, Y. Xu, J. Castro, D. Landa-Silva, "Particle Swarm Optimization for the Steiner Tree in Graph and Delay-Constrained Multicast Routing Problems," Journal of Heuristics, 2012, doi: 10.1007/s10732-012-9198-2.

[32] C.W. Duin, S. Voß, "The pilot method: A strategy for heuristic repetition with application to the Steiner problem in graphs," Networks, Vol.34, 1999, pp.181-191.

[33] H. Höller, B. Melian, S. Voß, "Applying the pilot method to improve VNS and GRASP metaheuristics for the design of SDH/WDM networks," European Journal of Operational Research, Vol.191, No.3, 2008, pp.691-704.

[34] T.A. Feo, M.G.C. Resende, "Greedy randomized adaptive search procedures," Journal of Global Optimization, Vol.6, 1995, pp.109133.

[35] M.G.C. Resende, C.C. Ribeiro, "Greedy randomized adaptive search procedures," Glover F., Kochenberger G. (Eds.), Handbook of Metaheuristics, Kluwer Academic Publishers, Dordrecht, 2003, pp. 219-249.

[36] G. Kontoravdis, J.F. Bard, “A GRASP for the vehicle routing problem with time windows," ORSA Journal on Computing, Vol.7, 1995, pp.10-23.

[37] P.M. Pardalos, T. Qian, M.G.C. Resende, "A greedy randomized adaptive search procedure for the feedback vertex set problem," Journal of Combinatorial Optimization, Vol.2, 1999, pp.399-412.

[38] M.G.C. Resende, L.S. Pitsoulis, P.M. Pardalos, "Fortran subroutines for computing approximate solutions of weighted MAX-SAT problems using GRASP," Discrete Applied Mathematics, Vol.100, 2000, pp.95-113.

[39] N. Mladenovic, P. Hansen, "Variable neighborhood search," Computers \& Operations Research, Vol.24, 1997, pp.1097-1100.

[40] D.P. Bertsekas, J.N. Tsitsiklis, C. Wu, "Rollout algorithms for combinatorial optimization," Journal of heuristics, Vol.3, 1997, pp.245-262.

[41] C. Meloni, D. Pacciarelli, M. Pranzo, "A Rollout Metaheuristic for Job Shop Scheduling Problems," Annals of Operations Research, Vol.131, 2004, pp.215-235.

[42] S. Voß, A. Fink, C.W. Duin, "Looking ahead with the pilot method," Annals of Operations Research. Vol.136, 2005, pp. 285-302.

[43] D. Eppstein, "Finding the $\mathrm{k}$ shortest paths," SIAM J. Computing.Vol.28,1998,pp.652-673. 